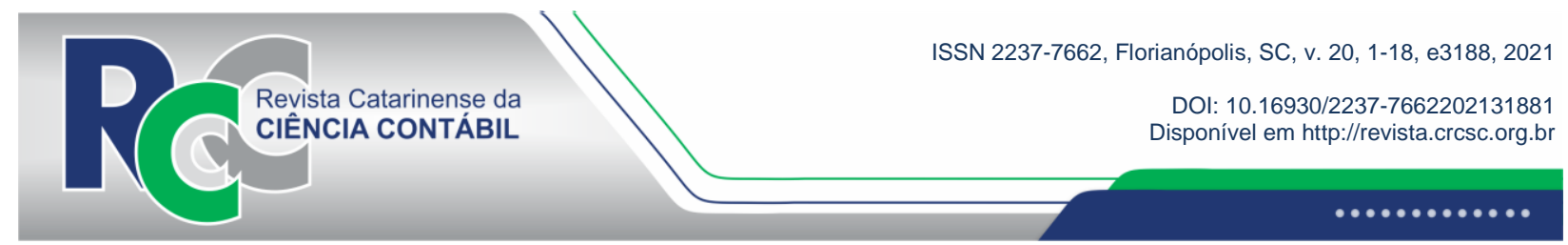

\title{
LIQUIDEZ ACIONÁRIA E AGRESSIVIDADE FISCAL NO MERCADO DE CAPITAIS BRASILEIRO
}

\author{
EQUITY LIQUIDITY AND TAX AGGRESSIVENESS IN THE BRAZILIAN \\ CAPITAL MARKET
}

\author{
BERNARDO FERNANDES LOTT PRÍMOLA \\ Universidade Federal de Minas Gerais. Endereço: Av. Presidente Antônio \\ Carlos, 6627| Pampulha | 31270-090 | Belo Horizonte/MG | Brasil. \\ (D) https://orcid.org/0000-0002-6028-2156 \\ bernardoflprimola@gmail.com
}

\section{EDUARDO MENDES NASCIMENTO}

Universidade Federal de Minas Gerais. Endereço: Av. Presidente Antônio Carlos, 6627 | Pampulha | 31270-090 | Belo Horizonte/MG | Brasil.

(D) http://orcid.org/0000-0002-2188-9748

e.mn@uol.com.br

\section{OCTÁVIO VALENTE CAMPOS}

Universidade Federal de Minas Gerais. Endereço: Av. Presidente Antônio Carlos, 6627| Pampulha | 31270-090 | Belo Horizonte/MG | Brasil.

(D) https://orcid.org/0000-0002-0354-0796

octaviovc@yahoo.com.br

\section{RESUMO}

Este estudo investigou a potencial relação entre a liquidez acionária e a agressividade fiscal no mercado de capitais brasileiro. Utilizando uma base de dados de companhias brasileiras de capital aberto do período de 2010 a 2019 - não incluindo o ano de 2020 pelos efeitos atípicos da pandemia da Covid-19 - foram desenvolvidos modelos de dados em painel cuja metassíntese consistiu em avaliar os efeitos longitudinais da liquidez acionária, variável independente, sobre o book tax difference, variável dependente e proxy da agressividade fiscal. Os resultados apontaram uma relação estatisticamente significante e economicamente positiva entre a proxy de agressividade fiscal e a liquidez acionária. O resultado sugere que empresas com ações menos voláteis, com maiores participações relativas em negócios da B3 e menor custo de negociação tendem a adotar um planejamento tributário mais agressivo. Este artigo contribui para demonstrar que em um mercado de capitais emergente, como o brasileiro, os investidores tendem a menosprezar com parcimônia a aumentos pontuais dos lucros por práticas tributárias mais agressivas, contudo, que

Editado em português e inglês. Versão original em português.

Pesquisa financiada pela Fundação de Amparo à Pesquisa do Estado de Minas Gerais - FAPEMIG.

Recebido em 16/7/2021. Revisado em 30/9/2021. Aceito em 13/10/2021 pelos Prof. Dr. Sérgio Murilo Petri (Editor-Chefe) e Prof. Dr. Sandro Vieira Soares (Editor Adjunto). Publicado em 12/11/2021.

Copyright (C) 2021 RCCC. Todos os direitos reservados. É permitida a citação de parte de artigos sem autorização prévia, desde que identificada a fonte. 
possam resultar em prejuízos futuros. Outrossim, o presente estudo contribui para demonstrar a importância das divulgações sobre planejamentos tributários, a fim de que os agentes de mercado possam adequadamente apreçar os ativos financeiros.

Palavras-chave: Agressividade Fiscal. Liquidez Acionária. Assimetria Informacional. Divulgação.

\begin{abstract}
This study has investigated the potential relationship between equity liquidity and tax aggressiveness in the Brazilian capital market. Using a database of publicly traded Brazilian companies from 2010 to 2019 - not including the year 2020 due to the atypical effects of the COVID-19 pandemic - panel data models have been developed, the goal synthesis of which consisted in evaluating the longitudinal effects of equity liquidity, independent variable, on the book tax difference, dependent variable, and proxy of tax aggressiveness. Results have shown a statistically significant and economically positive relationship between the tax aggressiveness proxy and stockholding liquidity. Results suggests that companies with less volatile stocks, with larger relative stocks in B3 [(in full, B3 - Brasil Bolsa Balcão S.A.), formerly BM\&FBOVESPA, a stock exchange located at São Paulo, Brazil] businesses and lower trading costs tend to adopt a more aggressive tax planning. This article helps to demonstrate that in an emerging capital market such as the Brazilian one investor tend to belittle occasional increases in profits sparingly through more aggressive tax practices, however, which may result in future losses. Furthermore, this study helps to demonstrate the importance of disclosures about tax planning so that market agents can properly price financial assets.
\end{abstract}

Keywords: Tax Aggressiveness. Equity Liquidity. Informational asymmetry. Disclosure.

\title{
1 INTRODUÇÃO
}

O principal objetivo deste estudo foi avaliar potenciais correlações entre a liquidez das companhias brasileiras de capital aberto e a proxy de agressividade fiscal. Isso porque, segundo Hanlon e Heitzman (2010), a agressividade fiscal é definida como uma redução explícita na carga tributária sobre o lucro. É oportuno estabelecer de antemão que o conceito de agressividade fiscal não se confunde com a ideia de evasão fiscal, posto que a essência desse conceito decorre da execução de atividades estritamente legais e de planejamento eficientes, os quais provocam a redução da carga tributária explicita, incrementando os retornos empresariais (Chen, Chen, Cheng, \& Shevlin, 2010; Scholes, Wolfson, Erickson, Maydew, \& Shevlin, 2014).

Outrossim, as diferentes proxies de agressividade fiscal podem capturar efeitos naturais das próprias práticas e procedimentos contábeis e fiscais que não se relacionam com planejamentos. Enquanto algumas proxies evidenciam apenas o planejamento tributário, outras capturam também o gerenciamento de resultados. Essa diferença ocorre por conta da inconsistência existente entre a contabilidade financeira e a contabilidade fiscal, as quais possuem arcabouços diferentes para o tratamento de determinadas questões pontuais (Formigoni, Antunes \& Paulo, 2009; Hanlon \& Heitzman, 2010; Martinez \& Leal, 2019).

Ressalvadas as delimitações acima, tem-se que o estudo da carga tributária sobre o lucro das empresas de capital aberto é atividade oportuna, pois, ainda que reduções da tributação sobre o lucro possam aumentar os lucros líquidos, a literatura prévia tem mapeado que os investidores tendem a analisar com cautela tais incrementos pela possibilidade de associação da agressividade 
fiscal com a elevação da assimetria informacional entre agentes internos e externos (Wahab \& Holland, 2012).

Além disso, Wahab e Holland (2012) indicam que a agressividade fiscal, em algumas ocasiões, pode decorrer de planejamentos ou condutas da administração fiscal que não são integralmente divulgados nos relatórios corporativos. Portanto, a agressividade fiscal pode resultar em maiores dificuldades para se projetar os fluxos de caixa de uma empresa; bem como, ocultar determinados riscos de autuações por parte das autoridades fiscais (Cao \& Wan, 2014).

Nesse contexto, é esperado que os agentes de mercado monitorem as corporações de capital aberto visando obter informações relevantes para apreçar os instrumentos patrimoniais acerca dos planejamentos tributários, de modo que esse acompanhamento seja maior para ações com maior liquidez (Bebchuk \& Weisbach, 2010; Chen, Ge, Louis, \& Zolotoy, 2019).

No âmbito do mercado de capitais brasileiro é possível verificar uma ampliação dos estudos avaliando potenciais práticas de agressividade fiscal, mais especificamente, sob a presença de diferentes níveis de alavancagem financeira (Martinez \& Martins, 2016); considerando a relação com partes relacionadas (Martinez \& Dalfior, 2015); bem como, tomando como referência idiossincrasias dos sistemas de governança corporativa (França \& Monte, 2019).

Posto isso, o presente estudo visou responder o seguinte problema de pesquisa: Como a liquidez de ações influencia a agressividade fiscal das empresas brasileiras de capital aberto listadas na B3 (Brasil, Bolsa e Balcão)? Dessa maneira, essa pesquisa possui o objetivo de avaliar potenciais práticas sinalizações de agressividade fiscal vis-à-vis à liquidez acionária das empresas brasileiras de capital aberto.

Este estudo se justifica por três motivos. O primeiro se refere à alta volatilidade dos mercados de capitais de países emergentes, como o mercado brasileiro (Lahrech \& Sylwester, 2011; Al Nasser \& Hajilee, 2016; Mclver \& Kang, 2020). E, por isso, é importante atentar-se para as sinalizações que podem influenciar essa volatilidade.

O segundo motivo se dá pelo papel da liquidez das ações como forma de minimização da assimetria informacional. A liquidez das ações pode possuir um efeito de vigilância na política fiscal da empresa, ao fiscalizar possíveis práticas abusivas de agressividade fiscal, isto é, a evasão fiscal (Cao \& Wan, 2014; Chen et al., 2019). A liquidez das ações melhora a informatividade no preço das ações, porque o valor da intervenção dos acionistas na gestão da empresa, e consequentemente, na físcalização das políticas fiscais, é refletido nos preços das ações mais rapidamente quando a ação é líquida, podendo ainda ocasionar o aumento do valor da ação. Isto gera um ciclo, ao qual os acionistas percebem os benefícios dessa intervenção quando as ações são mais líquidas, o que incentiva ainda mais as intervenções dos acionistas (Chen \& Zolotoy, 2014; Chen et al., 2019).

A terceira justificativa desse estudo é relativa às distintas características dos impostos diretos no Brasil, dentre as quais: possui maior complexidade do sistema tributário; maior vantagem competitiva pela não adoção de full disclosure; permissão de uso de pagamento de juros sobre o capital próprio como dedutibilidade da base de cálculo do IRPJ; dentre outros que podem, inclusive, incentivar a evasão fiscal (Almeida Júnior, 2007; Santos, Carvalho \& Ávila, 2019).

Outrossim, os resultados deste estudo reforçam a importância da ampla divulgação de práticas de gestão fiscal, posto que tais divulgações têm o condão de reduzir eventuais planejamentos tributários mais arrojados, os quais, embora possam resultar em incremento dos lucros presentes, podem culminar com reduções futuras dos fluxos de caixa. Essa redução se dá por conta de autuações fiscais e custos de consultores jurídicos, para mitigar potenciais decorrências dessas autuações fiscais (Hanlon \& Heitzman, 2010; Blaylock, Shelvin \& Wilson, 2012; Vello \& Martinez, 2014). 


\section{REVISÃO DE LITERATURA E DESENVOLVIMENTO DE HIPÓTESE}

\subsection{Agressividade fiscal e suas distinções do conceito de evasão fiscal}

Uma empresa possui diversos instrumentos que podem ser empregados de modo a diminuir sua carga tributária (Martinez \& Dalfior, 2015). Por essa razão, é fundamental distinguir o que é planejamento fiscal e o que é evasão fiscal, que é um ato, inclusive, proibido por leis nacionais. Dessa forma, a agressividade fiscal não implica ilegalidade e em muitos casos ela é até mesmo desejada e incentivada.

Hanlon e Heitzman (2010) definem a agressividade fiscal como uma redução explícita dos tributos sobre o lucro. Nessa linha, Lisowsky, Robinson e Schmidt (2013) caracterizam a agressividade fiscal como atividades na extremidade do continuum de minimização tributária. Slemrod e Yitzhaki (2002) ressaltam que a característica distintiva entre agressividade fiscal e evasão fiscal é a ilegalidade desse último conceito.

Ainda a respeito da evasão fiscal, Frischmann, Shevlin e Wilson (2008) a definem como o engajamento em posições fiscais significativas com fatos comprobatórios relativamente fracos. Já quanto à agressividade fiscal, adotando o entendimento de que ela se trata de uma atividade totalmente legal, Chen et al. (2010) a definem como uma redução gerencial da renda tributável por meio de ações de planejamento tributário.

Importa lembrar que o planejamento tributário envolve um conjunto de fatores que auxiliam na redução dos tributos explícitos, desde que aplicados dentro das práticas de governança corporativa e sob um arcabouço de legalidade (Vello \& Martinez, 2014; Tôrres, 2001).

Scholes et al. (2014) sustentam que o planejamento tributário decorre de um conjunto de ações visando promover a redução dos tributos explícitos, não acrescendo outros custos ou tributos com efeitos marginais superiores às reduções alcançadas, gerando, assim, maior eficiência tributária.

Há alguns anos, o Brasil implementou dispositivos antielisivos no Código Tributário Nacional, pelos quais as autoridades fiscais têm conseguido combater e desestimular os atos de elisão fiscal artificial ou elusiva, dos contribuintes (Martinez \& Ramalho, 2017).

\subsection{Liquidez de ações}

A liquidez das ações é uma medida multidimensional que possui diversas características transacionais, tais como: tightness, depth, resiliency, immediacy e breadth (Kyle, 1985; Amihud, 2002; Sarr \& Lybek, 2002; Lesmond, 2005; Menezes da Silva, 2009; Vieira, Justen Junior, \& Righi, 2015). Essa abrangência multidimensional propiciou o emprego de diferentes métricas, das quais algumas são relacionadas à atividade de negociação (turnover), enquanto outras são vinculadas aos valores de negociação (bid-ask spread) (Machado \& Medeiros, 2012).

Holmström e Tirole (1993) estudaram o valor do mercado de ações como monitor de desempenho gerencial. Eles identificaram que o bid-ask spread é determinado apenas pela frequência de negociação dos traders de liquidez, ou seja, traders de longo prazo não tendem a influenciar o desconto nos preços à vista.

Bhattacharya, Desai e Venkataraman (2013) investigaram a associação entre qualidade dos ganhos acionários e a assimetria informacional em uma amostra de empresas da NYSE (New York Stock Exchange) e da NASDAQ (National Association of Securities Dealers Automated Quotations) no período de 1998 a 2007. Os autores identificaram que a baixa qualidade dos ganhos está significativamente associada a uma maior assimetria de informação.

Menezes da Silva (2009) identificou a relação entre liquidez das ações e o nível de divulgação das empresas brasileiras de capital aberto no setor de siderurgia e de metalurgia no período entre 1998 e 2007. As variáveis de liquidez e a divulgação da informação (disclosure) 
apresentam relação positiva em geral. $\mathrm{O}$ autor indicou que é preferível investir em ações de empresas que mantenham uma política de divulgação de informações voltadas para a diminuição da assimetria de informação. Em linhas gerais, o desconhecimento dado pela falta de informação resulta em menor liquidez de ações e maior custo de capital.

Outro fator que afeta a liquidez das ações é o papel de insider trading (Chung, Goh, Lee, \& Shevlin, 2019). Jayaraman e Milbourn (2012) investigaram se o papel da liquidez das ações influencia a composição do salário anual do CEO e a sensibilidade da riqueza gerencial aos preços das ações. $\mathrm{O}$ estudo apontou que as empresas com maior liquidez das ações dependem menos da remuneração dos gestores baseada em caixa como parte da remuneração total dos contratos anuais. Adicionalmente, concluíram que a dependência dos preços das ações na elaboração da remuneração executiva é maior para empresas com maior liquidez.

No âmbito específico do mercado de capitais brasileiro, Machado e Medeiros (2012) analisaram a existência por meio das seguintes variáveis: turnover, volume negociado, quantidade de negócios, negociabilidade (liquidez em bolsa) e turnover padronizado. Machado e Medeiros (2012) concluíram que existe um "prêmio" de liquidez no mercado brasileiro e que as políticas corporativas podem suavizar os custos de liquidez, isto é, elas melhoram a liquidez dos títulos reduzindo o custo de capital e aumentando o valor da empresa.

Essas políticas corporativas possuem, também, o objetivo de fornecer mais informações voluntárias ao mercado, reduzindo a assimetria de informações e o risco percebido pelas partes interessadas (Amihud \& Mendelson, 2006). Dessa forma, os principais podem se beneficiar dessas novas informações, adotando-as como mecanismo de vigilância dos agentes.

Posto isso, é possível conjecturar a seguinte hipótese:

$H_{1}$ A liquidez de mercado inibe as práticas de agressividade fiscal à medida que é requerido um maior volume informacional sobre a administração fiscal das empresas com maior liquidez acionária.

\section{ABORDAGEM METODOLÓGICA}

\subsection{Dados e Amostra do estudo}

Os dados utilizados neste estudo foram obtidos na plataforma Economatica ${ }^{\circledR} \mathrm{e}$ compreendem as empresas brasileiras não financeiras listadas na B3 no período entre 2010 e 2019 . Esse período foi escolhido devido ao fato de 2009 ter sido o último ano após o período de transição das normas contábeis brasileiras para o padrão internacional. Segundo Martinez e Silva (2017), essa mudança favoreceu o efeito da comparabilidade entre os demonstrativos contábeis das empresas.

Outrossim, não foi incluído o ano de 2020 em decorrência da pandemia da Covid-19, cujos efeitos sobre as séries temporais de mercados financeiros, sobretudo, por conta das paralisações econômicas, que ainda estão sendo assimilados e compreendidos. As empresas financeiras foram excluídas devido às suas características e particularidades em relação à legislação tributária (Famá \& French, 1992; Machado \& Medeiros, 2012; França \& Monte, 2019).

As observações com ausência de dados e os outliers foram excluídos, respectivamente. Os outliers foram identificados utilizando-se o Método do Quartil na variável dependente, conforme descrito por Oliveira, Caroli, Amaral e Vilca (2014). Em seguida, aplicou-se winsorização a 5\%, conforme descrito por Tukey (1962). Ao final, resultaram 1304 observações provenientes de 232 empresas. 


\subsection{Variáveis}

Nesse estudo, optou-se pelo uso do Book-Tax Diferences (BTD) como métrica representativa da variável dependente Agressividade Fiscal, especialmente por essa ser uma métrica amplamente utilizada pela literatura, que busca analisar o comportamento de agressividade fiscal, bem como por essa métrica conseguir capturar distinções normativas do lucro contábil visà-vis o lucro fiscal (Carvalho, Paulo \& Tavares, 2014).

Mills (1998) define Book-Tax Diferences (BTD) como a receita contábil antes dos impostos menos a receita tributável, ou seja, o lucro contábil deduzido o lucro tributável.

$$
B T D_{i t}=L A I R_{i t}-\left(\frac{I R / C S L L_{i t}}{0,34}\right)
$$

Compulsando a variável dependente BTD aos demais termos preditores do seu comportamento estatístico, é possível estabelecer o modelo para teste da Hipótese (1), tal como segue na Equação (2) a seguir.

$$
\begin{gathered}
\text { BTD }_{i t}=\alpha+\beta_{1} \cdot \text { Vol }_{i t}+\beta_{2} \cdot \text { Liqb }_{i t}+\beta_{3} \cdot \text { Qneg }_{i t}+\beta_{4} \cdot \text { Qtit }_{i t}+\beta_{5} \cdot \text { Turn }_{1 t}+\beta_{6} \cdot \text { Tur }_{i t}+\beta_{7} \cdot \operatorname{Sprd}_{i t} \\
+ \text { Variáveis de Controle }+\varepsilon_{i t}
\end{gathered}
$$

As variáveis independentes de liquidez das ações - termos de interesse - estão representadas na Tabela 1. A variável Liquidez não é diretamente observável e apresenta diversos aspectos que não podem ser capturados por uma única medida. Por isso, a fim de testar a hipótese de pesquisa, decidiu-se utilizar variáveis que capturem a multidimensionalidade da liquidez das ações (Amihud, 2002; Menezes da Silva, 2009; Vieira et al., 2015).

\begin{tabular}{|c|c|c|c|c|}
\hline Nome & Símbolo & Descrição & $\begin{array}{c}\text { Sinal } \\
\text { Esperado }\end{array}$ & Referência \\
\hline $\begin{array}{l}\text { Volume } \\
\text { Financeiro }\end{array}$ & VOL & $\begin{array}{l}\text { Volume financeiro de um } \\
\text { ativo negociado, no período } \\
\text { de um ano }\end{array}$ & $(-)$ & $\begin{array}{l}\text { Brennan, Chordia e Subrahmanyam (1998); } \\
\text { Lesmond (2005); Vieira e Milach (2008); } \\
\text { Menezes da Silva (2009); Vieira et al. } \\
\text { (2015); Machado e Medeiros (2012); } \\
\text { Perobelli, Famá e Sacramento (2016); } \\
\text { Silveira, Vieira, Bender Filho e Coronel } \\
\text { (2017). }\end{array}$ \\
\hline $\begin{array}{l}\text { Liquidez em } \\
\text { bolsa }\end{array}$ & LIQB & $100 x\left(\frac{p}{P}\right) x \sqrt{\frac{n}{N} x \frac{v}{V}}$ & $(-)$ & $\begin{array}{l}\text { Menezes da Silva (2009); Machado e } \\
\text { Medeiros (2012); Martins e Paulo (2014); } \\
\text { Silva, Nardi, Martins e Barossi Filho (2016). }\end{array}$ \\
\hline $\begin{array}{l}\text { Quantidade } \\
\text { de negócios }\end{array}$ & QNEG & $\begin{array}{l}\text { Número de ocorrências de } \\
\text { compra e venda de um ativo } \\
\text { em um ano }\end{array}$ & $(-)$ & $\begin{array}{l}\text { Vieira e Milach (2008); Menezes da Silva } \\
\text { (2009); Machado e Medeiros (2012); Vieira, } \\
\text { Justen Junior e Righi (2015); Silveira et al. } \\
\text { (2017). }\end{array}$ \\
\hline $\begin{array}{l}\text { Quantidade } \\
\text { de títulos }\end{array}$ & QTIT & $\begin{array}{c}\text { Quantidade de ações } \\
\text { negociadas em um ano }\end{array}$ & $(-)$ & $\begin{array}{l}\text { Vieira e Milach (2008); Menezes da Silva } \\
\text { (2009); Silveira et al. (2017). }\end{array}$ \\
\hline Turnover 1 & TUR1 & $\frac{\text { QTIT }}{\text { Preço médio da } a c ̧ a ̃ o} o_{t}$ & $(-)$ & $\begin{array}{l}\text { Vieira e Milach (2008); Menezes da Silva } \\
\text { (2009); Perobelli, Famá e Sacramento } \\
\text { (2016). }\end{array}$ \\
\hline Turnover 2 & TUR2 & $\frac{\text { QTIT }}{\text { Qtd de ações emitidas }}$ & $(-)$ & $\begin{array}{l}\text { Datar, Naik e Radcliffe (1998); Menezes da } \\
\text { Silva (2009); Vieira et al. (2015); Perobelli, } \\
\text { Famá e Sacramento (2016); Silveira et al. } \\
\text { (2017). }\end{array}$ \\
\hline
\end{tabular}

Tabela 1

\section{Variáveis independentes de interesse}




\begin{tabular}{c|c|c|c|c|c}
$\begin{array}{c}\text { Spread } \\
\text { máximo e } \\
\text { mínimo }\end{array}$ & SPRD & $100 x\left(\frac{\text { Preço Máx-Preço Min }}{\text { Preço Médio }}\right)$ & $(+)$ & $\begin{array}{l}\text { Vieira e Milach (2008); Menezes da Silva } \\
(2009) ; \text { Vieira } \text { et al. (2015); Perobelli, Famá } \\
\text { e Sacramento (2016); Silva et al. (2016). }\end{array}$ \\
\hline
\end{tabular}

Legenda: $\mathrm{p}=$ número de dias em que houve pelo menos um negócio da ação; $\mathrm{P}=$ número total de dias; $\mathrm{n}=$ número de negócios com a ação; $\mathrm{N}$ = número de negócios com todas as ações; $\mathrm{v}=$ volume em dinheiro com a ação; $\mathrm{V}=$ volume em dinheiro com todas as ações; QTIT = quantidade de ações negociadas em um ano.

As variáveis de controle estão na Tabela 2. A escolha dessas variáveis foi concebida com base em estudos anteriores, os quais também avaliaram a liquidez acionária, todavia com objetivos distintos dos explorados neste estudo.

Tabela 2

\section{Variáveis de controle}

\begin{tabular}{|c|c|c|c|c|}
\hline Nome & Símbolo & Descrição & $\begin{array}{c}\text { Sinal } \\
\text { Esperado }\end{array}$ & Referência \\
\hline Disponibilidades & CASH & $\frac{{\text { Caixa e Equiv. } \text { Caixa }_{t}}_{\text {Ativo } \text { Total }_{t-1}}}{\text { A }}$ & + & $\begin{array}{l}\text { Chen e Zolotoy (2014); Chen et al. } \\
\text { (2019); Chiachio e Martinez (2019). }\end{array}$ \\
\hline $\begin{array}{l}\text { Resultado de } \\
\text { Equivalência } \\
\text { Patrimonial }\end{array}$ & EQUIV & $\frac{\text { Equiv. Patrimonial }_{t}}{{\text { Ativo } \text { Total }_{t-1}}}$ & -+ & $\begin{array}{l}\text { Nakao (2012); McGuire, Wang e } \\
\text { Wilson (2014); Chen e Zolotoy } \\
\text { (2014); Brunozi, Kronbauer, } \\
\text { Martinez \& Alves (2018); Chen } \text { et al. } \\
\text { (2019). }\end{array}$ \\
\hline $\begin{array}{l}\text { Tamanho da } \\
\text { Empresa }\end{array}$ & SIZE & $\ln (\text { Ativo Total })_{t}$ & + & $\begin{array}{l}\text { Rego (2003); Chen e Zolotoy (2014); } \\
\text { Cao e Wan (2014); Gaertner (2014); } \\
\text { Martins e Paulo (2014); Chen e Lin } \\
\text { (2017); Chen et al. (2019); Chiachio e } \\
\text { Martinez (2019); França e Monte } \\
\text { (2019). }\end{array}$ \\
\hline Ativos Intangíveis & INTAG & $\frac{\text { Ativos Intangíveis }_{t}}{{\text { Ativo } \text { Total }_{t-1}}}$ & +- & $\begin{array}{l}\text { Chen e Zolotoy (2014); Cao e Wan } \\
\text { (2014); Gaertner (2014); Chen et al. } \\
\text { (2019); Chiachio e Martinez (2019); } \\
\text { Melo, Castro Moraes, Souza e } \\
\text { Nascimento (2020). }\end{array}$ \\
\hline Alavancagem & ALAV & $\frac{{\text { Passivo Não } \text { Circulante }_{t}}_{\text {Ativo } \text { Total }_{t-1}}}{\text { At }}$ & + & $\begin{array}{l}\text { Chen e Zolotoy (2014); Cao e Wan } \\
\text { (2014); Gaertner (2014); Chen et al. } \\
\text { (2019); Chiachio e Martinez (2019); } \\
\text { França e Monte (2019); Moraes et al. } \\
\text { (2021). }\end{array}$ \\
\hline Ativo Imobilizado & IMOB & 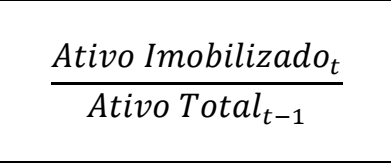 & + & $\begin{array}{l}\text { Chen e Zolotoy (2014); Cao e Wan } \\
\text { (2014); Chen e Lin (2017); Chen et al. } \\
\text { (2019); Chiachio e Martinez (2019); } \\
\text { Melo et al. (2020). }\end{array}$ \\
\hline Retorno dos Ativos & ROA & $\frac{\text { LAIR }_{t}}{\text { Ativo Total }_{t-1}}$ & + & $\begin{array}{l}\text { McGuire et al. (2014); Chen e } \\
\text { Zolotoy (2014); Cao e Wan (2014); } \\
\text { Gaertner (2014); Chen et al. (2019); } \\
\text { Chiachio e Martinez (2019); França e } \\
\text { Monte (2019). }\end{array}$ \\
\hline $\begin{array}{c}\text { Variação do Retorno } \\
\text { dos Ativos }\end{array}$ & SROA & $R O A_{t}-R O A_{t-1}$ & - & $\begin{array}{l}\text { Chen e Zolotoy (2014); Gaertner } \\
\text { (2014); Chen et al. (2019). }\end{array}$ \\
\hline
\end{tabular}

Legenda: - Disponibilidades (CASH): definido como Caixa e Equivalente de Caixa do ano dividido pelo Ativo total do ano anterior, por ser uma variável de controle para nível de caixa da empresa; - Resultado de Equivalência Patrimonial (EQUIV): definido como o Resultado de Equivalência Patrimonial do ano dividido pelo Ativo total do ano anterior. Controla as atividades de investimentos das empresas (extraídos das demonstrações consolidadas); Tamanho da Empresa (SIZE): utiliza-se o logaritmo natural do Ativo total do ano. Controla a política de custos do Tax Avoidance; - Ativos Intangíveis (INTAG): intangíveis totais do ano dividido pelo Ativo total do ano anterior. Controla, juntamente com IMOB, a capacidade da empresa em proteger a renda por meio da depreciação e da amortização; - Alavancagem (ALAV): total do Passivo Não Circulante do ano dividido pelo Ativo total do ano 
anterior. Controla o efeito da dívida de longo prazo; - Ativo Imobilizado (IMOB): total de Imobilizados no ano dividido pelo Ativo total do ano anterior. Controla, juntamente com INTAG, a capacidade da empresa em proteger a renda por meio da depreciação e da amortização; - Retorno dos Ativos (ROA): definido como o LAIR do ano dividido pelo Ativo total do ano anterior. Controla a lucratividade da empresa; - Variação do Retorno de Ativos (SROA): ROA do ano atual menos o ROA do ano anterior dividido pelo Ativo total do ano anterior. Controla, juntamente com ROA, a lucratividade e incertezas na operação da empresa.

Em prol do robustecimento estatístico dos parâmetros estimados nesse material, a Equação (2) foi estimada por meio das diferentes abordagens existentes acerca dos modelos de dados em painel. Desse modo, visando-se determinar qual dos modelos de regressão é o mais ajustado, foram empregados os testes de Chow, de Hausman e de LM de Breusch-Pagan.

\section{APRESENTAÇÃO E ANÁliSE dOS RESULTADOS}

As principais estatísticas descritivas se encontram descritas na Tabela 3. Dentre as principais métricas para caracterização da distribuição da variável BTD se destaca o coeficiente de Kurtoisis ( $\mathrm{k}>3)$, o qual indica que a variável BTD é Leptocúrtica. Essa constatação dá sinais de um efeito de cauda longa (Chissom, 1970). Esse resultado pode indicar que as empresas com proxies mais agressivas ou conservadoras podem ter impactos significativos pela concentração de maior severidade.

Já o coeficiente de Skewness $(\mathrm{v}<0)$ indica que a cauda esquerda é mais pesada e que, portanto, há uma assimetria nos dados. Isso aponta que há um maior número de empresas no primeiro quartil da amostra. As variáveis Índice de Liquidez em Bolsa, Quantidade de Negócios e Quantidade de Títulos possuem comportamentos estatísticos semelhantes ao se analisar a distribuição amostral. O Índice de Liquidez em Bolsa, por sua vez, apresenta mediana e a média muito próximas, 0,0123 e 0,1395 , o que indica maior normalidade na distribuição amostral. Contudo, a variabilidade dos dados na amostragem continua elevada, uma vez que o desvio padrão é de 0,2496 e a amplitude Q3-Q1 é de 0,1549.

A análise das variáveis Quantidade de Negócios e Quantidade de Títulos conduz a um resultado semelhante. A média e a mediana de ambas as variáveis apresentam um valor próximo: 333.683,30 e 34.870,00, respectivamente, para a Quantidade de Negócios e 144.549,10 e 17.731,47, respectivamente, para a variável Quantidade de Títulos.

Ao se comparar os valores das medidas de tendência central com os valores de amplitude, percebe-se que há uma concentração de dados mais próxima dos valores de Q1 do que dos valores de Q3. Ao se analisar os valores das médias e das medianas com os valores de máximo e de mínimo, encontra-se uma nuvem de dados muito mais próxima dos mínimos do que dos máximos.

Tabela 3

\section{Estatística descritiva dos dados}

\begin{tabular}{|c|c|c|c|c|c|c|c|c|c|}
\hline Var. & Média & $\begin{array}{l}\text { Desvio } \\
\text { Padrão }\end{array}$ & Min & Max & Q1 & Q2 & Q3 & Skewness & Curtose \\
\hline BTD & $13 \mathrm{mil}$ & $130 \mathrm{mil}$ & $-440 \mathrm{mil}$ & $340 \mathrm{mil}$ & $-26 \mathrm{mil}$ & 7,6 mil & $70 \mathrm{mil}$ & $-0,53$ & 4,79 \\
\hline Cash & 0,08 & 0,09 & - & 0,39 & 0,01 & 0,05 & 0,12 & 1,58 & 5,28 \\
\hline Equiv & - & - & $-0,01$ & 0,01 & - & - & - & 0,95 & 9,08 \\
\hline Size & 14,27 & 1,69 & 9,85 & 17,23 & 13,3 & 14,41 & 15,46 & $-0,51$ & 2,96 \\
\hline Intag & 0,15 & 0,2 & - & 0,78 & - & 0,04 & 0,23 & 1,53 & 4,41 \\
\hline Alav & 0,42 & 0,4 & 0,03 & 2,23 & 0,18 & 0,33 & 0,52 & 2,83 & 12,47 \\
\hline Imob & 0,26 & 0,23 & & 0,87 & 0,04 & 0,22 & 0,4 & 0,83 & 2,85 \\
\hline
\end{tabular}




\begin{tabular}{|c|c|c|c|c|c|c|c|c|c|}
\hline ROA & 0,06 & 12,43 & $-48,9$ & 18,49 & $-2,15$ & 2,49 & 6,51 & $-2,09$ & 8,47 \\
\hline SROA & - & - & - & - & - & - & - & - & 13,51 \\
\hline VOL & $2.100 \mathrm{mil}$ & $4.100 \mathrm{mil}$ & 240 & $18.000 \mathrm{mil}$ & $11 \mathrm{mil}$ & 160.000 & $2.000 \mathrm{mil}$ & 2,58 & 9,28 \\
\hline LIQB & 0,14 & 0,25 & - & 1,02 & - & 0,01 & 0,15 & 2,16 & 6,93 \\
\hline QNEG & $330 \mathrm{mil}$ & 580.000 & 59 & $2.400 \mathrm{mil}$ & 2,3 mil & $35 \mathrm{mil}$ & 400 mil & 2,12 & 6,84 \\
\hline QTIT & $140 \mathrm{mil}$ & $270 \mathrm{mil}$ & 6,4 & $1.200 \mathrm{mil}$ & 900 & $18 \mathrm{mil}$ & $160 \mathrm{mil}$ & 2,53 & 9,03 \\
\hline TUR1 & $18 \mathrm{mil}$ & $36 \mathrm{mil}$ & 0,15 & $170 \mathrm{mil}$ & 110 & $2,5 \mathrm{mil}$ & $16 \mathrm{mil}$ & 2,91 & 11,26 \\
\hline TUR2 & 0,58 & 0,64 & - & 2,74 & 0,08 & 0,37 & 0,85 & 1,53 & 5,05 \\
\hline SPRD & 0,84 & 0,73 & 0,22 & 3,65 & 0,4 & 0,58 & 0,94 & 2,31 & 8,21 \\
\hline
\end{tabular}

Esse resultado demonstra, mais uma vez, que há preferência por negociar certas ações no mercado brasileiro e que essas ações possuem grandes quantidades de títulos, grandes quantidades de negócios e movimentam um alto volume financeiro, típico de um mercado concentrado.

Por fim, a variável Quantidade de Negócios apresenta alta multicolinearidade ao nível de significância de 1\% com a variável Quantidade de Títulos, 0,8353 corroborando os resultados encontrados por Menezes da Silva (2009), fortalecendo a tese de que a liquidez é multidimensional e que não é diretamente observável, sendo necessárias múltiplas medidas para tal (Amihud, 2002; Menezes da Silva, 2009; Vieira et al., 2015).

Tabela 4

Análise de correlação de Pearson

\begin{tabular}{|c|c|c|c|c|c|c|c|c|c|c|c|c|c|c|c|c|}
\hline Var. & 1 & 2 & 3 & 4 & 5 & 6 & 7 & 8 & 9 & 10 & 11 & 12 & 13 & 14 & 15 & 16 \\
\hline $1 \mathrm{BTD}$ & 1 & & & & & & & & & & & & & & & \\
\hline $2 \mathrm{CASH}$ & $\underline{0,08}$ & 1 & & & & & & & & & & & & & & \\
\hline 3 EQUIV & $\underline{0,1}$ & 0,03 & 1 & & & & & & & & & & & & & \\
\hline 4 SIZE & $\underline{0,13}$ & $\underline{0,16}$ & $0,06^{* *}$ & 1 & & & & & & & & & & & & \\
\hline 5 INTAG & $\underline{0,08}$ & $\underline{0,11}$ & $-0,02$ & $\underline{0,3}$ & 1 & & & & & & & & & & & \\
\hline 6 ALAV & $\underline{-0,14}$ & $-0,02$ & $-0,03$ & $\underline{-0,23}$ & 0,01 & 1 & & & & & & & & & & \\
\hline 7 IMOB & $-0,04$ & 0,02 & $-0,05^{*}$ & 0,01 & $\underline{-0,28}$ & $\underline{0,17}$ & 1 & & & & & & & & & \\
\hline $8 \mathrm{ROA}$ & $\underline{0,46}$ & $\underline{0,23}$ & $0,07 * *$ & $\underline{0,37}$ & $\underline{0,19}$ & $\underline{-0,36}$ & $-0,06^{* *}$ & 1 & & & & & & & & \\
\hline 9 SROA & 0,04 & 0,03 & $-0,01$ & 0,01 & 0,02 & $0,07 * *$ & 0,03 & $\underline{0,25}$ & 1 & & & & & & & \\
\hline $10 \mathrm{VOL}$ & $\underline{0,14}$ & $\underline{0,16}$ & $\underline{0,07}$ & $\underline{0,52}$ & $\underline{0,2}$ & $-0,05^{*}$ & $-0,03$ & $\underline{0,2}$ & 0 & 1 & & & & & & \\
\hline $11 \mathrm{LIQB}$ & $\underline{0,14}$ & $\underline{0,16}$ & $\underline{0,07}$ & $\underline{0,55}$ & $\underline{0,2}$ & $-0,05^{*}$ & $-0,03$ & $\underline{0,21}$ & 0 & $\underline{0,96}$ & 1 & & & & & \\
\hline 12 QNEG & $\underline{0,11}$ & $\underline{0,15}$ & $\underline{0,09}$ & $\underline{0,56}$ & $\underline{0,19}$ & $-0,05^{*}$ & $-0,05^{* *}$ & $\underline{0,19}$ & 0 & $\underline{0,89}$ & $\underline{0,95}$ & 1 & & & & \\
\hline 13 QTIT & $\underline{0,15}$ & $\underline{0,15}$ & $\underline{0,14}$ & $\underline{0,49}$ & $\underline{0,15}$ & $-0,01$ & $-0,04$ & $\underline{0,15}$ & 0 & $\underline{0,81}$ & $\underline{0,84}$ & $\underline{0,83}$ & 1 & & & \\
\hline 14 TUR1 & $\underline{0,07}$ & $\underline{0,1}$ & $\underline{0,15}$ & $\underline{0,37}$ & $0,06^{* *}$ & 0 & $\underline{-0,08}$ & $\underline{0,08}$ & 0 & $\underline{0,48}$ & $\underline{0,55}$ & $\underline{0,58}$ & $\underline{0,84}$ & 1 & & \\
\hline 15 TUR2 & $-0,05^{*}$ & $-0,01$ & $-0,02$ & $\underline{0,13}$ & $0,05^{*}$ & 0,03 & $\underline{-0,16}$ & $\underline{-0,07}$ & 0,01 & $\underline{0,43}$ & $\underline{0,46}$ & $\underline{0,48}$ & $\underline{0,4}$ & $\underline{0,35}$ & 1 & \\
\hline 16 SPRD & $\underline{-0,3}$ & $\underline{-0,19}$ & $-0,06^{* *}$ & $\underline{-0,29}$ & $\underline{-0,13}$ & $\underline{0,19}$ & $-0,04$ & $\underline{0,46}$ & 0,01 & $\underline{-0,16}$ & $\underline{-0,16}$ & $\underline{-0,16}$ & $\underline{-0,15}$ & $-0,06^{* *}$ & $\underline{0,26}$ & 1 \\
\hline
\end{tabular}

Legenda: [sublinhado] = Significância a 1\%; [**] = Significância a 5\%; [*] = Significância a 10\%.

Em relação ao modelo mais adequado, o Teste de Chow indicou o modelo de efeitos fixos $(\mathrm{F}=4,26$, Prob $>\mathrm{F}=0,000)$. $\mathrm{O}$ Teste de Hausman corroborou a indicação do modelo de efeitos fixos $($ Chi2 $=12,90$, Prob $>$ chi2 $=0,2292)$. Por fim, o Teste de LM de Breusch-Pagan indicou a predileção do modelo de efeitos aleatórios (chibar2 $=238,13$, Prob $>$ chibar2 $=0,0000)$. Em função 
das indicações cruzadas a respeito do melhor formato de estimação dos modelos de dados em painel, optou-se pela aplicação de ambas as técnicas, efeitos fixos e efeitos aleatórios (Tabela 5).

O Modelo de Efeitos Fixos com Cluster apresenta como resultado do Teste F o valor de 6,4830, enquanto o Modelo de Efeitos Fixos apresenta o valor de 23,3524 (Tabela 5). Com base nos resultados dos testes $\mathrm{F}$ conclui-se que a melhor abordagem de estimação é a técnica de efeitos fixos.

Tabela 5

Comparação dos Modelos de Dados em Painel

\begin{tabular}{|c|c|c|c|c|c|c|}
\hline Var. & POOL & PROBS & $\mathrm{EF}$ & EFCLUST & EA & EACLUS \\
\hline CASH & $\begin{array}{r}-67.890,32 \\
(35.050,29)\end{array}$ & $\begin{array}{r}-67.890,32 \\
(45.084,83)\end{array}$ & $\begin{array}{r}-51.886,47 \\
(44.989,35) \\
\end{array}$ & $\begin{array}{r}-51.886,47 \\
(71.342,26)\end{array}$ & $\begin{array}{r}-67.649,63 \\
(39.554,31)\end{array}$ & $\begin{array}{r}-67.649,63 \\
(55.245,60) \\
\end{array}$ \\
\hline EQUIV & $\begin{array}{r}2.350 .622,70 \\
(946.350,47)\end{array}$ & $\begin{array}{r}2.350 .622,70 \\
(14.24 .967,50)\end{array}$ & $\begin{array}{r}3.309 .387,30 \\
(1.009 .618,10)\end{array}$ & $\begin{array}{r}3.309 .387,30 \\
(1.346 .898,50)\end{array}$ & $\begin{array}{l}3.112 .367,50 \\
(935.775,33)\end{array}$ & $\begin{array}{r}3.112 .367,50 \\
(1.289 .642,30)\end{array}$ \\
\hline SIZE & $\begin{array}{r}-7.473,14 \\
(2.528,28) \\
\end{array}$ & $\begin{array}{r}-7.473,14 \\
(4.191,05)\end{array}$ & $\begin{array}{l}-21.758,15 \\
(9.164,18) \\
\end{array}$ & $\begin{array}{r}-21.758,15 \\
(12.608,07)\end{array}$ & $\begin{array}{r}-9.868,00 \\
(4.157,36) \\
\end{array}$ & $\begin{array}{r}-9.868,00 \\
(5.206,38)\end{array}$ \\
\hline INTAG & $\begin{array}{r}-7.576,09 \\
(17.167,07) \\
\end{array}$ & $\begin{array}{r}-7.576,09 \\
(26.539,57)\end{array}$ & $\begin{array}{r}-26.444,28 \\
(41.231,40)\end{array}$ & $\begin{array}{r}-26.444,28 \\
(62.636,02)\end{array}$ & $\begin{array}{r}-12.250,11 \\
(25.305,19)\end{array}$ & $\begin{array}{r}-12.250,11 \\
(30.028,98) \\
\end{array}$ \\
\hline ALAV & $\begin{array}{l}12.390,21 \\
(8.861,43) \\
\end{array}$ & $\begin{array}{r}12.390,22 \\
(13.277,47)\end{array}$ & $\begin{array}{r}16.440,59 \\
(14.824,39) \\
\end{array}$ & $\begin{array}{r}16.440,59 \\
(18.248,25) \\
\end{array}$ & $\begin{array}{r}19.558,21 \\
(11.464,29) \\
\end{array}$ & $\begin{array}{r}19.558,21 \\
(14.091,28) \\
\end{array}$ \\
\hline IMOB & $\begin{array}{r}-13.627,13 \\
(14.527,36)\end{array}$ & $\begin{array}{r}-13.627,13 \\
(21.005,30)\end{array}$ & $\begin{array}{r}5.747,18 \\
(32.767,11) \\
\end{array}$ & $\begin{array}{r}5.747,18 \\
(42.364,22) \\
\end{array}$ & $\begin{array}{r}-9.807,63 \\
(21.465,59)\end{array}$ & $\begin{array}{r}-9.807,63 \\
(24.348,99) \\
\end{array}$ \\
\hline ROA & $\begin{array}{l}4.967,23 \\
(335,76)\end{array}$ & $\begin{array}{l}4.967,23 \\
(684,82)\end{array}$ & $\begin{array}{l}5.322,41 \\
(362,28)\end{array}$ & $\begin{array}{l}5.322,41 \\
(835,34)\end{array}$ & $\begin{array}{l}5.329,01 \\
(335,55)\end{array}$ & $\begin{array}{l}5.329,01 \\
(755,74)\end{array}$ \\
\hline SROA & $\begin{array}{r}-1,3 \mathrm{E}+08 \\
(4,4 \mathrm{E}+07)\end{array}$ & $\begin{array}{r}-1,3 \mathrm{E}+08 \\
(5,7 \mathrm{E}+07)\end{array}$ & $\begin{array}{r}-1,3 \mathrm{E}+08 \\
(3,8 \mathrm{E}+07)\end{array}$ & $\begin{array}{r}-1,3 \mathrm{E}+08 \\
(6,1 \mathrm{E}+07)\end{array}$ & $\begin{array}{r}-1,3 \mathrm{E}+08 \\
(3,7 \mathrm{E}+07)\end{array}$ & $\begin{array}{r}-1,3 \mathrm{E}+08 \\
(6,0 \mathrm{E}+07)\end{array}$ \\
\hline VOL & $\begin{array}{r}-0,00 \\
(0,00)\end{array}$ & $\begin{array}{l}-0,00 \\
(0,01)\end{array}$ & $\begin{array}{r}-0,00 \\
(0,00)\end{array}$ & $\begin{array}{r}-0,00 \\
(0,00)\end{array}$ & $\begin{array}{r}-0,00 \\
(0,00) \\
\end{array}$ & $\begin{array}{r}-0,00 \\
(0,00)\end{array}$ \\
\hline LIQB & $\begin{array}{r}84.615,48 \\
(67.557,67) \\
\end{array}$ & $\begin{array}{r}84.615,48 \\
(116.869,81) \\
\end{array}$ & $\begin{array}{r}121.598,58 \\
(71.283,30) \\
\end{array}$ & $\begin{array}{r}121.598,58 \\
(124.598,22) \\
\end{array}$ & $\begin{array}{r}99.427,32 \\
(63.008,95) \\
\end{array}$ & $\begin{array}{r}99.427,32 \\
(104.572,91)\end{array}$ \\
\hline QNEG & $\begin{array}{l}-0,05 \\
(0,02) \\
\end{array}$ & $\begin{array}{l}-0,05 \\
(0,03)\end{array}$ & $\begin{array}{l}-0,01 \\
(0,02)\end{array}$ & $\begin{array}{l}-0,01 \\
(0,04) \\
\end{array}$ & $\begin{array}{r}-0,03 \\
(0,02) \\
\end{array}$ & $\begin{array}{r}-0,03 \\
(0,03)\end{array}$ \\
\hline QTIT & $\begin{array}{r}0,16 \\
(0,04) \\
\end{array}$ & $\begin{array}{r}0,16 \\
(0,06) \\
\end{array}$ & $\begin{array}{r}-0,04 \\
(0,06) \\
\end{array}$ & $\begin{array}{r}-0,04 \\
(0,09) \\
\end{array}$ & $\begin{array}{r}0,13 \\
(0,05) \\
\end{array}$ & $\begin{array}{r}0,13 \\
(0,07) \\
\end{array}$ \\
\hline TUR1 & $\begin{array}{r}-0,54 \\
(0,21) \\
\end{array}$ & $\begin{array}{r}-0,54 \\
(0,33)\end{array}$ & $\begin{array}{l}-0,85 \\
(0,24)\end{array}$ & $\begin{array}{l}-0,85 \\
(0,38) \\
\end{array}$ & $\begin{array}{r}-0,78 \\
(0,22) \\
\end{array}$ & $\begin{array}{r}-0,78 \\
(0,36)\end{array}$ \\
\hline TUR2 & $\begin{array}{r}-835,65 \\
(6.242,12) \\
\end{array}$ & $\begin{array}{r}-835,64 \\
(9.854,28) \\
\end{array}$ & $\begin{array}{r}12.345,07 \\
(8.308,59)\end{array}$ & $\begin{array}{r}12.345,07 \\
(11.851,40)\end{array}$ & $\begin{array}{r}1.246,52 \\
(7.020,43) \\
\end{array}$ & $\begin{array}{r}1.246,51 \\
(9.719,84)\end{array}$ \\
\hline SPRD & $\begin{array}{r}-17.646,63 \\
(5.262,56) \\
\end{array}$ & $\begin{array}{r}-17.646,63 \\
(5.954,10) \\
\end{array}$ & $\begin{array}{r}-14.881,67 \\
(5.081,60) \\
\end{array}$ & $\begin{array}{r}-14.881,67 \\
(5.494,84) \\
\end{array}$ & $\begin{array}{r}-15.924,06 \\
(4.847,58) \\
\end{array}$ & $\begin{array}{r}-15.924,06 \\
(5.358,17) \\
\end{array}$ \\
\hline _cons & $\begin{array}{r}134.058,97 \\
(35.903,94) \\
\end{array}$ & $\begin{array}{r}134.058,97 \\
(58.501,09) \\
\end{array}$ & $\begin{array}{r}336.721,57 \\
(128.528,69) \\
\end{array}$ & $\begin{array}{r}336.721,57 \\
(178.645,90) \\
\end{array}$ & $\begin{array}{r}165.118,77 \\
(58.630,21) \\
\end{array}$ & $\begin{array}{r}165.118,77 \\
(71.737,81) \\
\end{array}$ \\
\hline $\mathrm{N}$ & $1.304,0$ & $1.304,00$ & $1.304,00$ & $1.304,00$ & $1.304,00$ & $1.304,00$ \\
\hline $\mathrm{R}^{2}$ & 0,2544 & 0,2544 & 0,2489 & 0,2489 & - & - \\
\hline $\mathrm{R}^{2} \mathrm{O}$ & - & - & 0,1322 & 0,1322 & 0,2445 & 0,2445 \\
\hline $\mathrm{R}^{2} \mathrm{~B}$ & - & - & 0,0423 & 0,0423 & 0,1971 & 0,1971 \\
\hline $\mathrm{R}^{2} \mathrm{~W}$ & - & - & 0,2489 & 0,2489 & 0,2368 & 0,2368 \\
\hline
\end{tabular}




\begin{tabular}{c|rrrrrr} 
F & 29,29 & 7,95 & 23,35 & 6,48 & - & - \\
Q2 & - & - & - & - & 391,30 & 109,51 \\
sigma_u & - & - & $114.809,35$ & $114.809,35$ & $84.166,52$ & 84166,52 \\
sigma_e & - & - & $90.031,39$ & $90.031,39$ & $90.031,39$ & $90.031,39$ \\
rho theta & - & - & 0,6192 & 0,6192 & 0,4664 & 0,4664 \\
\hline
\end{tabular}

Legenda: $\mathrm{POOL}=$ Pooled; PROBS $=$ Pooled com Cluster; $\mathrm{EF}=$ Efeitos Fixos; EFCLUST $=$ Efeitos Fixos com Cluster; $\mathrm{EA}=$ Efeitos Aleatórios; EACLUS $=$ Efeitos Aleatórios com Cluster.

As variáveis não significativas do modelo foram removidas com o propósito de atenuar possíveis efeitos da multicolinearidade no resultado, através da técnica Stepwise (Fávero, 2015). A técnica consiste na subtração gradativa das variáveis que apresentaram pior resultado no p-valor. Adotou-se, como significativo para o modelo, p-valor ao nível de significância a 1\%, resultando no modelo final apresentado na Tabela 6.

A autocorrelação foi verificada mediante o Teste de Wooldridge, o qual apresentou $\mathrm{F}(1,172)$ de 0,920 e Prob $>\mathrm{F}$ de 0,3387 . Com base nesse resultado não se aceita a hipótese nula de ausência de autocorrelação.

Esse modelo apresentou um coeficiente de determinação $\left(\mathrm{R}^{2}\right)$ de 0,1535 ao nível de significância de $1 \%$. Esse resultado demonstra que a liquidez das ações possui relação com as proxies de agressividade fiscal das empresas do mercado acionário brasileiro. Esse resultado é condizente com o estudo de Chen et al. (2019), que encontrou um valor similar de $\mathrm{R}^{2}(0,149) \mathrm{em}$ uma Regressão OLS e valores entre 0,16 e 0,23 para os quartis entre $10 \%$ e $40 \%$ em uma Análise de Regressão de Quartil.

Tabela 6

\section{Preditores significativos no Modelo de Efeitos Fixos}

\begin{tabular}{l|rrrrrr}
\multicolumn{1}{c}{ VAR. } & \multicolumn{1}{c}{ COEF } & \multicolumn{1}{c}{ STD. ERR } & \multicolumn{1}{c}{$\mathrm{T}$} & $\mathrm{P}>|\mathrm{t}|$ & \multicolumn{2}{c}{$95 \%$ Intervalo de Confiança } \\
\hline EQUIV & $3.146 .476,00$ & $995.158,80$ & 3,16 & 0,002 & $1.193 .781,00$ & $5.099 .170,00$ \\
SIZE & $-25.833,58$ & $8.398,82$ & $-3,08$ & 0,002 & $-42.309,78$ & $-9357,38$ \\
ROA & $5.263,98$ & 351,78 & 14,96 & 0,000 & $4.573 .706,00$ & $5.954,25$ \\
SROA & $-1,21 \mathrm{e}+08$ & $3,76 \mathrm{e}+07$ & $-3,23$ & 0,001 & $-1,95 \mathrm{e}+08$ & $-4,77 \mathrm{e}+07$ \\
LIQB & $86.101,25$ & $30.140,70$ & 2,86 & 0,004 & $26.959,25$ & $145.243,20$ \\
TUR1 & $-0,87$ & 0,18 & $-4,80$ & 0,000 & $-1,23$ & $-0,52$ \\
SPRD & $-13.317,87$ & $4.953,39$ & $-2,69$ & 0,007 & $-23.037,39$ & $-3.598,34$ \\
CONST & $394.503,90$ & $118.958,80$ & 3,32 & 0,001 & $161.083,7$ & $627.924,10$ \\
\hline
\end{tabular}

\begin{tabular}{lclr}
\hline $\mathrm{N}$ & 1.304 & $\mathrm{Rho}$ & 0,6059 \\
$\mathrm{R}^{2}$ within & 0,2448 & $\mathrm{~F}(6,1066)$ & 49,32 \\
$\mathrm{R}^{2}$ between & 0,0695 & $\mathrm{Prob}>\mathrm{F}$ & 0,00 \\
$\mathrm{R}^{2}$ overall & 0,1535 & corr $\left(\mathrm{u} \_\mathrm{i}, \mathrm{Xb}\right)$ & $-0,2430$ \\
\hline \multicolumn{2}{l}{ Teste de Autocorrelação (Wooldridge) } & \multicolumn{2}{c}{ Teste de Heterocedasticidade (Wald) } \\
$\mathrm{F}(1,172)$ & 0,920 & Chi2 $(232)$ & $6,4 \mathrm{e}+31$ \\
Prob $>\mathrm{F}$ & 0,3387 & Prob $>$ Chi2 & 0,0000 \\
\hline
\end{tabular}

\subsection{Análise das variáveis de controle}

A variável Equivalência Patrimonial (EQUIV) apresentou coeficiente de 3.146.476, desvio-padrão de 995.158,80 é significante no modelo, segundo o Teste-t, de 3,16 ao nível de significância de 1\%. Essa variável que controla as atividades de investimentos das companhias em 
empresas controladas e coligadas se comportou conforme o esperado para o mercado brasileiro, conforme Nakao (2012) e Brunozi et al. (2018).

A variável Tamanho da Empresa (SIZE) apresentou coeficiente de -25.833,58, desviopadrão de 8.398,82 e representatividade no modelo, segundo o Teste-t, de $-3,08$ ao nível de significância de $1 \%$. O comportamento da variável contraria os determinados pelos estudos de Rego (2003), Chen e Zolotoy (2014), Cao e Wan (2014), Gaertner (2014), Chen e Lin (2017), Chen et al. (2019), Chiachio e Martinez (2019), França e Monte (2019). É possível que os gestores de companhias menores, com políticas de custo mais frágeis, aproveitam o foco dos investidores nas grandes companhias, com políticas de custo mais desenvolvidas, para adotarem práticas mais acentuadas de agressividade fiscal e potencializar os seus resultados.

A variável de controle Retorno dos Ativos (ROA) é representativa no modelo, conforme o Teste-t, com o valor de 14,96 ao nível de significância de 1\%. Seu coeficiente de 5.263,98 e seu desvio-padrão de 351,78 indicam que um aumento na lucratividade aumenta a agressividade fiscal. Esse resultado está em concordância com a premissa de relação positiva entre essas duas variáveis, segundo os trabalhos de McGuire et al. (2014), Chen e Zolotoy (2014), Cao e Wan (2014), Gaertner (2014), Chen et al. (2019), Chiachio e Martinez (2019), França e Monte (2019).

A variável Variação do Retorno dos Ativos (SROA) apresentou coeficiente de $-1,21 \mathrm{e}+08$, desvio-padrão de 3,76e+07 e representatividade no modelo, segundo o Teste-t, de -3,23 ao nível de significância de 1\%. A variável se comportou como esperado, conforme os estudos de Chen e Zolotoy (2014), Gaertner (2014), e Chen et al. (2019). O coeficiente indica que há uma relação negativa entre a variável SROA e a agressividade fiscal. Como a variável SROA indica a lucratividade e a incerteza das operações, pode-se interpretar que quanto menor a variação da lucratividade, menor o grau de incerteza das operações e maior a agressividade fiscal. Isso parece ser evidente, pois a agressividade fiscal é uma excelente ferramenta para auxiliar na potencialização do resultado, pois mantendo a lucratividade constante ela torna as ações atrativas para os investidores.

\subsection{Análise de variáveis representativas da liquidez de ações}

O resultado da regressão da Tabela 6 indica que a liquidez das ações influencia as práticas de agressividade fiscal. De forma geral, o resultado indica que quanto mais líquida é a ação de uma empresa, mais agressivo é o seu planejamento tributário. Esse resultado rejeita a hipótese de que a liquidez de mercado inibe as práticas de agressividade fiscal à medida em que é requerido um maior volume informacional sobre a administração fiscal das empresas com maior liquidez acionária.

Por ser um constructo multidimensional, as principais características da liquidez de ações que influenciaram a agressividade fiscal foram: volatilidade (Turnover 1), Negociabilidade (Índice de Liquidez em Bolsa) e o Custo de Negociação Instantânea (Bid-ask spread). As variáveis Índice de Liquidez em Bolsa e Bid-ask spread apresentaram um comportamento contrário ao esperado.

A variável Turnover 1 (TUR1) apresentou coeficiente de $-0,87$ e desvio-padrão de 0,18 . Essa variável foi a mais representativa no modelo, com valor de $-4,80$ baseando-se no Teste-t, ao nível de significância de 1\%. Esses resultados sugerem que o efeito mitigador da liquidez das ações na agressividade fiscal é atenuado para empresas com altos níveis de volatilidade sobre as ações. Esse corolário corrobora os resultados propostos por Cao e Wan (2014), e Chen et al. (2019). Assim, as empresas que possuem ações menos voláteis no mercado tendem a apresentar um planejamento tributário mais agressivo.

Já a variável Î́ndice de Liquidez em Bolsa (LIQB) apresentou coeficiente de 86.101,25 e desvio padrão de 30.140,70. Essa variável demonstra relevância no modelo de 2,86 com base no Teste-t ao nível de significância de $1 \%$. Esse resultado indica que as empresas que apresentam 
melhores índices de negociabilidade na bolsa de valores, isto é, que possuem maior participação relativa de sua ação em negócios conduzidos na B3, adotam um planejamento tributário mais agressivo.

A variável Bid-ask spread (SPRD), por sua vez, possui coeficiente angular de $-13.317,87$ e desvio-padrão de 4.953,39. Esta variável é significativa no modelo, segundo o Teste-t, a -2,69, cujo p-valor é de $1 \%$. O resultado indica que, as ações de empresas que apresentam menor custo de negociação imediata, possuem maior agressividade fiscal. Essa métrica representa o custo de seleção adversa, o qual consiste em negociações com grande quantidade de ações, e que possuem o poder de gerar quedas ou aumento no preço das ações (Menezes da Silva, 2009). Essa situação tende a ocorrer quando novas informações usadas pelo investidor ainda não foram refletidas no preço de mercado das ações, ou seja, o negociador pode ser detentor de informações privilegiadas (Menezes da Silva, 2009).

O comportamento do Bid-ask spread pode indicar que os gestores aproveitam que alguns negociadores possuem informações privilegiadas e assim potencializam o resultado da empresa por meio do planejamento tributário agressivo, maximizando também sua remuneração atrelada à performance (dessa forma menosprezam o custo de agência).

De forma geral, os gestores das empresas que possuem ações pouco voláteis, com maiores participações relativas em negócios da B3 e menor custo de negociação, tendem a adotar um planejamento tributário mais agressivo. Os resultados sugerem que a liquidez de ações no mercado acionário brasileiro não possui papel repressor da assimetria de informação. Além disso, os principais das empresas do mercado acionário brasileiro parecem menosprezar o risco potencial de autuações fiscais, ao passo da alta negociabilidade e potencial maximização dos resultados das empresas.

\section{CONSIDERAÇÕES FINAIS}

O monitoramento dos acionistas é uma ferramenta necessária para inibir práticas abusivas dos gestores, diminuir a assimetria informacional e trazer mais segurança aos investidores. Neste contexto, Chen et al. (2019) descobriram que as ações com maior liquidez são de empresas que possuem maiores níveis de acionistas ativistas, que proporcionam seguridade aos demais investidores, mitigando a assimetria informacional e inibindo a agressividade fiscal em seus níveis extremos.

Nesse contexto, este estudo investigou a relação existente entre liquidez de ações e agressividade fiscal nas empresas abertas no mercado acionário brasileiro entre os anos de $2010 \mathrm{e}$ 2019. Observamos indícios de que a liquidez de ações influencia práticas de agressividade fiscal e que as empresas que possuem ações mais líquidas adotam práticas mais agressivas de planejamento tributário. Esse comportamento é contrário aos encontrados por Cao e Wan (2014) e Chen et al. (2019).

Além disso, o nosso resultado sugere que empresas com ações menos voláteis, com maiores participações relativas em negócios da B3 e menor custo de negociação, tendem a adotar um planejamento tributário mais agressivo. Além disso, o comportamento da variável Bid-ask spread parece indicar que alguns negociadores de ações podem possuir informações privilegiadas. Segundo Menezes da Silva (2009), esse resultado pode indicar que novas informações, usadas pelo investidor, ainda não foram refletidas no preço de mercado das ações, ou seja, o negociador pode ser detentor de informações privilegiadas.

Este estudo contribui para demonstrar que em um mercado de capitais emergente, como o brasileiro, os investidores tendem a menosprezar com parcimônia a aumentos pontuais dos lucros por práticas tributárias mais agressivas, contudo, que possam resultar em prejuízos futuros. Outrossim, o presente estudo contribui para demonstrar a importância das divulgações sobre 
planejamentos tributários, a fim de que os agentes de mercado possam adequadamente apreçar os ativos financeiros.

Esta pesquisa está limitada à investigação da existência de uma relação entre agressividade fiscal e liquidez de ações e dos fatores que possam imbuir essa relação. Para trabalhos futuros, sugerimos que a agressividade fiscal seja testada com a liquidez de ações em análises por quartil de acordo com os níveis de agressividade fiscal e por tipo de ação, ordinária e preferencial.

\section{REFERÊNCIAS}

Almeida Júnior, E. S. (2007). Sistema tributário, governança corporativa e abertura de capital: Brasil versus Estados Unidos. Dissertação de Mestrado, Universidade Federal de Uberlândia.

Amihud, Y. (2002). Illiquidity and stock returns: cross-section and time-series effects. Journal of Financial Markets, 5(1), 31-56.

Al Nasser, O. M., \& Hajilee, M. (2016). Integration of emerging stock markets with global stock markets. Research in International Business and Finance, 36, 1-12.

Amihud, Y., \& Mendelson, H. (2006). Stock and bond liquidity and its effect on prices and financial policies. Financial Markets and Portfolio Management, 20(1), 19-32.

Bebchuk, L. A., \& Weisbach, M. S. (2010). The state of corporate governance research. The review of financial studies, 23(3), 939-961.

Bhattacharya, N., Desai, H., \& Venkataraman, K. (2013). Does earnings quality affect information asymmetry? Evidence from trading costs. Contemporary Accounting Research, 30(2), 482-516.

Blaylock, B., Shevlin, T., \& Wilson, R. J. (2012). Tax avoidance, large positive temporary booktax differences, and earnings persistence. The Accounting Review, 87(1), 91-120.

Brennan, M. J., Chordia, T., \& Subrahmanyam, A. (1998). Alternative factor specifications, security characteristics, and the cross-section of expected stock returns. Journal of Financial Economics, 49(3), 345-373.

Brunozi, A. C., Kronbauer, C. A., Martinez, A. L., \& Alves, T. W. (2018). BTD anormais, accruals discricionários e qualidade dos accruals em empresas de capital aberto listadas no Brasil. Revista Contemporânea de Contabilidade, 15(35), 108-141.

Cao, S., \& Wan, C. (2014). Stock liquidity and tax avoidance: Evidence from natural experiments.

Carvalho, V. G., Paulo, E., \& Tavares, A. D. L. (2014). Gerenciamento tributário: proxies utilizadas pelas pesquisas no período 2000 a 2012. Registro Contábil, 5(3), 1-19.

Chen, S., Chen, X., Cheng, Q., \& Shevlin, T. (2010). Are family firms more tax aggressive than non-family firms?. Journal of financial economics, 95(1), 41-61.

Chen, T., \& Lin, C. (2017). Does information asymmetry affect corporate tax aggressiveness?. Journal of Financial and Quantitative Analysis, 52(5), 2053-2081. 
Chen, Y., Ge, R., Louis, H., \& Zolotoy, L. (2019). Stock liquidity and corporate tax avoidance. Review of Accounting Studies, 24(1), 309-340.

Chen, Y., \& Zolotoy, L. (2014). Stock Liquidity and Corporate Tax-Avoidance: The Tale of Two Tails. School of Accounting Seminar Series, Kensington, NSW, Australia.

Chiachio, V. F. D. O., \& Martinez, A. L. (2019). Efeitos do Modelo de Fleuriet e Índices de Liquidez na Agressividade Tributária. Revista de Administração Contemporânea, 23(2).

Chissom, B. S. (1970). Interpretation of the kurtosis statistic. The American Statistician, 24(4), 1922.

Chung, S. G., Goh, B. W., Lee, J., \& Shevlin, T. (2019). Corporate tax aggressiveness and insider trading. Contemporary Accounting Research, 36(1), 230-258.

Datar, V. T., Naik, N. Y., \& Radcliffe, R. (1998). Liquidity and stock returns: An alternative test. Journal of Financial Markets, 1(2), 203-219.

Famá, E. F., \& French, K. R. (1992). The Cross-section of expected stock returns. Journal of Finance, 47(2), 427-465.

Fávero, L. P. (2015). Análise de dados. (1ª ed.) Rio de Janeiro. Elsevier.

Formigoni, H., Antunes, M. T. P., \& Paulo, E. (2009). Diferença entre o lucro contábil e lucro tributável: uma análise sobre o gerenciamento de resultados contábeis e gerenciamento tributário nas companhias abertas brasileiras. BBR-Brazilian Business Review, 6(1), 44-61.

França, R. D. de, \& Monte, P. A. do (2019). Efeitos da remuneração, governança e reputação corporativa na tax avoidance de empresas brasileiras de capital aberto sob a ótica do oportunismo gerencial. Anais do USP International Conference in Accounting, São Paulo.

Frischmann, P. J., Shevlin, T., \& Wilson, R. (2008). Economic consequences of increasing the conformity in accounting for uncertain tax benefits. Journal of Accounting and Economics, 46(2-3), 261-278.

Gaertner, F. B. (2014). CEO after-tax compensation incentives and corporate tax avoidance. Contemporary Accounting Research, 31(4), 1077-1102.

Hanlon, M., \& Heitzman, S. (2010). A Review of tax research. Journal of Accounting and Economics, 50(2-3), 127-178.

Holmström, B., \& Tirole, J. (1993). Market liquidity and performance monitoring. Journal of Political Economy, 101(4), 678-709.

Jayaraman, S., \& Milbourn, T. T. (2012). The Role of stock liquidity in executive compensation. The Accounting Review, 87(2), 537-563.

Kyle, A. S. (1985). Continuous Auctions and Insider Trading. Econometrica, 53(3). 
Lahrech, A., \& Sylwester, K. (2011). US and Latin American stock market linkages. Journal of International Money and Finance, 30(7), 1341-1357.

Lesmond, D. A. (2005). Liquidity of emerging markets. Journal of Financial Economics, 77(2), 411-452.

Lisowsky, P., Robinson, L. A., \& Schmidt, A. P. (2013). Do Publicly disclosed tax reserves tell us about privately disclosed tax shelter activity?. Journal of Accounting Research, 51(3).

Machado, M. A. V., \& Medeiros, O. R. (2012). Existe o efeito liquidez no mercado acionário Brasileiro? BBR-Brazilian Business Review, 9(4), 28-51.

Martins, O. S., \& Paulo, E. (2014). Assimetria de informação na negociação de ações, características econômico-financeiras e governança corporativa no mercado acionário brasileiro. Revista Contabilidade \& Finanças, 25(64), 33-45.

Martinez, A. L., \& Leal, L. V. (2019). Conformidade contábil-fiscal e gerenciamento de resultados contábeis no Brasil. RACE-Revista de Administração, Contabilidade e Economia, 18(1), 9-30.

Martinez, A. L., \& Silva, R. F. da (2017). Agressividade Fiscal e o Custo de Capital de Terceiros no Brasil. Revista de Gestão, Finanças e Contabilidade, 7(1), 240-251.

Martinez, A. L., \& Dalfior, M. D. (2015). Agressividade fiscal entre companhias controladoras e controladas. Revista da Receita Federal: Estudos tributários e aduaneiros, 2(1), 344-362.

Martinez, A. L., \& Martins, V. A. M. (2016). Alavancagem financeira e agressividade fiscal no Brasil. Revista de Contabilidade da UFBA, 10(3), 5-22.

Martinez, A. L., \& Ramalho, V. P. (2017). Agressividade tributária e sustentabilidade empresarial no Brasil. Revista Catarinense da Ciência Contábil, 16(49).

McGuire, S. T., Wang, D., \& Wilson, R. J. (2014). Dual class ownership and tax avoidance. The Accounting Review, 89(4), 1487-1516.

Mclver, R. P., \& Kang, S. H. (2020). Financial crises and the dynamics of the spillovers between the US and BRICS stock markets. Research in International Business and Finance, 54, 101276.

Melo, L. Q., Castro Moraes, G. S., Souza, R. M., \& Nascimento, E. M. (2020). A responsabilidade social corporativa afeta a agressividade fiscal das firmas? Evidências do mercado acionário brasileiro. Revista Catarinense da Ciência Contábil, 19.

Menezes da Silva, R. L. (2009). Divulgação de informações e liquidez de ações: Evidências do setor de Siderurgia e Metalurgia no Brasil. Dissertação de Mestrado, USP.

Mills, L. F. (1998). Book-tax differences and Internal Revenue Service adjustments. Journal of Accounting Research, 36(2), 343-356. 
Moraes, G. S. C., Nascimento, E. M., Soares, S. V. N., \& Prímola, B. F. L. (2021). Tax avoidance and tax disclosure: A study of Brazillian listed companies. Contextus - Contemporary Journal of Economics and Management, 19(13), 197-216.

Nakao, S. H. (2012). A adoção de IFRS e o legado da conformidade contábil-fiscal mandatória. Tese de Doutorado, Faculdade de Economia, Administração e Contabilidade de Ribeirão Preto, Universidade de São Paulo.

Oliveira, C. D., Caroli, A. A. de, Amaral, A. S., \& Vilca, O. L. (2014). Detecção de fraudes, anomalias e erros em análise de dados contábeis: Um estudo com base em outliers. Revista Eletrônica do Departamento de Ciências Contábeis \& Departamento de Atuária e Métodos Quantitativos (REDECA), 1(1), 102-127.

Perobelli, F. F. C., Famá, R., \& Sacramento, L. C. (2016). Relações entre liquidez e retorno nas dimensões contábil e de mercado no Brasil. Revista Contabilidade \& Finanças, 27(71).

Rego, S. O. (2003). Tax avoidance activities of US multinational corporations. Contemporary Accounting Research, 20(4), 805-833.

Santos, M. V., Carvalho, H. L. M., \& Ávila, L. A. C. (2019). Complexidade Tributária e Evasão Fiscal no Brasil. Anais do Congresso USP de Iniciação Científica em Contabilidade, São Paulo, 16

Sarr, A., \& Lybek, T. (2002). Measuring liquidity in financial markets. International Monetary Fund, 2002(232), 1-63.

Scholes, M. S., Wolfson, M. A., Erickson, M., Maydew, E., \& Shevlin, T. (2014). Taxes \& business strategy. Upper Saddle River: Prentice Hall.

Silva, R. L. M. da, Nardi, P. C. C., Martins, V. A., \& Barossi Filho, M. (2016). Os níveis de governança corporativa da BM\&F BOVESPA aumentam a liquidez das ações?. Revista Base (Administração e Contabilidade) da UNISINOS, 13(3), 248-263.

Silveira, V. G. da, Vieira, K. M., Bender Filho, R., \& Coronel, D. A. (2017). Análise fatorial de séries temporais para medidas de liquidez no mercado brasileiro. RACE - Revista de Administração, Contabilidade e Economia, 16(3), 1109-1132.

Slemrod, J., \& Yitzhaki, S. (2002). Tax avoidance, evasion, and administration. In. A. J. Auerbach and F. Feldstein (eds.), Handbook of Public Economics (pp. 1423-1470).

Tôrres, H. T. (2001). Direito tributário internacional: Planejamento tributário e operações transnacionais. São Paulo: Revista dos Tribunais.

Tukey, J. W. (1962). The Future of data analysis. The Annals of mathematical statistics, 33(1), 167.

Vello, A. P. C., \& Martinez, A. L. (2014). Planejamento tributário eficiente: uma análise de sua relação com o risco de mercado. Revista Contemporânea de Contabilidade, 11(23). 
Vieira, K. M., Justen Júnior, A. A., \& Righi, M. B. (2015). O papel da liquidez e suas múltiplas dimensões no retorno das ações: Um estudo com dados em painel do mercado brasileiro. CONTEXTUS - Revista Contemporânea de Economia e Gestão, 13(2), 7-34.

Vieira, K. M., \& Milach, F. T. (2008). Liquidez/iliquidez no mercado brasileiro: Comportamento no período 1995-2005 e suas relações com o retorno. Revista Base, 5(1).

Wahab, N. S. A., \& Holland, K. (2012). Tax Planning, corporate governance and equity value. The British Accounting Review, 44(2), 111-124. 